\title{
Intracellular targeting of STIP1 inhibits human cancer cell line growth
}

\author{
Chiao-Yun Lin ${ }^{1,2 \#}$, Shun-Hua Chen ${ }^{1,2,3 \#}$, Chia-Lung Tsai ${ }^{4}$, Yun-Hsin Tang ${ }^{1,2}$, Kai-Yun Wu ${ }^{2}$, Angel Chao ${ }^{1,2}$ \\ ${ }^{1}$ Gynecologic Cancer Research Center, Linkou Chang Gung Memorial Hospital, Taoyuan; ${ }^{2}$ Department of Obstetrics and Gynecology, Linkou \\ Chang Gung Memorial Hospital and Chang Gung University, Taoyuan; ${ }^{3}$ Fooyin University School of Nursing, Kaohsiung; ${ }^{4}$ Genomic Medicine \\ Research Core Laboratory, Linkou Chang Gung Memorial Hospital, Taoyuan \\ Contributions: (I) Conception and design: SH Chen, A Chao, KY Wu; (II) Administrative support: A Chao ; (III) Provision of study materials: SH \\ Chen, CL Tsai, CY Lin; (IV) Collection and assembly of data: SH Chen, A Chao, YH Tang, CL Tsai; (V) Data analysis and interpretation: SH Chen, \\ A Chao, CL Tsai, KY Wu, CY Lin; (VI) Manuscript writing: All authors; (VII) Final approval of manuscript: All authors. \\ \#These authors contributed equally to this work. \\ Correspondence to: Angel Chao. Gynecologic Cancer Research Center, Linkou Chang Gung Memorial Hospital, Taoyuan. Email: drangiechao@gmail.com; \\ Kai-Yun Wu. 5, Fushin St, Guishan, Taoyuan. Email: m7030@cgmh.org.tw.
}

Background: Extracellular and cell-surface molecules remain the most common druggable cancer targets. However, intracellular therapeutic modalities are gaining momentum. The overexpression of stress-induced phosphoprotein 1 (STIP1), an adaptor protein that coordinates the functions of different chaperones in protein folding, has been reported in several solid malignancies. Here, we investigated the effects of intracellular STIP1 inhibition, attained either through the HEPES-mediated cytosolic delivery of antiSTIP1 antibodies or the use of a cell-penetrating signal-tagged peptide 520, in different human cancer cell lines and luciferase-expressing murine ovarian cancer cells (MOSEC/Luc) tumor-bearing C57BL/6 mice.

Methods: The effects of STIP1 in different human cell lines were determined by cell viability, cell cytotoxicity and cell apoptosis assays. Immunoblotting was used to assess the relevant proteins found in this study and tumor xenograft mice models were also employed.

Results: Intracellular targeting of STIP1 inhibited cancer cell line growth and promoted caspase 3-dependent apoptotic cell death. Moreover, the intracellular delivery of anti-STIP1 antibodies facilitated the degradation of STIP1 and two of its client proteins, lysine-specific demethylase 1 and Janus kinase 2. In vivo studies demonstrated that survival of mice bearing experimental tumors was improved by administration of anti-STIP1 antibodies.

Conclusions: Our findings demonstrate that the cytosolic inhibition of STIP1 in tumor cells is feasible and provides a solid basis for further investigation of STIP1 as an intracellular cancer target. Our findings demonstrate that cytosolic inhibition of STIP1 in tumor cells is feasible and provide a solid basis for further exploration of STIP1 as an intracellular cancer target.

Keywords: Intracellular targeting; cytosol; stress-induced phosphoprotein 1 (STIP1); cancer therapy; intracellular protein delivery

Submitted Nov 27, 2020. Accepted for publication Jan 27, 2021.

doi: $10.21037 /$ tcr-20-3333

View this article at: http://dx.doi.org/10.21037/tcr-20-3333

\section{Introduction}

Extracellular and cell-surface molecules remain the most common druggable cancer targets (1-3). However, the use of intracellular therapeutic modalities is gaining momentum
(4-6). In fact, a diverse group of oncoproteins involved in tumorigenesis are found in the cytoplasm and may serve as promising targets for novel anti-tumor therapeutic strategies $(4,7,8)$. 
Stress-induced phosphoprotein 1 (STIP1) is an adaptor protein that coordinates the functions of different chaperones, including heat shock proteins (HSPs), to facilitate protein folding within cells $(9,10)$. Accumulating evidence indicates that STIP1, which is overexpressed in several malignancies, including liver, pancreatic, ovarian, colon, and breast tumors, plays a role in the pathogenesis of cancer (11-20). Notably, STIP1 expression has been detected not only on the cell surface but also in the cytosol $(13,16)$, indicating both extracellular and intracellular STIP1 have oncogenic potential. In our previous study, we found that secreted STIP1 promoted the proliferation of ovarian cancer cells by activating the extracellular-regulated MAP kinases (ERK) or activin receptor-like kinase-2 (ALK2)-small mothers against the decapentaplegic (SMAD) signaling pathway $(13,21)$. Conversely, intracellular STIP1 promotes the growth of ovarian cancer cells through two distinct mechanisms - the formation of an HSP90janus kinase 2 (JAK2)-signal transducer and activator of transcription 3 (STAT3) complex and the glycogen synthase kinase-3 beta (GSK3ß)-mediated phosphorylation of lysine-specific demethylase 1 (LSD1) $(22,23)$. It has also been demonstrated that intracellular STIP1 mediates the heat-induced spread of hepatocellular carcinoma to distant sites by facilitating epithelial-mesenchymal transition via an increased nuclear shuttling of Snail1 (24). In addition, the exposure of cells to 4-(2-hydroxyethyl)1-piperazineethanesulfonic acid (HEPES) is an effective means to promote the intracellular delivery of either antibodies or small peptides (25).

The present study represents a proof-of-concept investigation to test whether intracellular STIP1 serves as a druggable target in different human cancer cell lines. Two different strategies have been used to attain intracellular STIP1 inhibition: (I) HEPES-induced cytosolic delivery of anti-STIP1 antibodies, and (II) exposure of cells to a cellpenetrating peptide (denoted as peptide 520) capable of inhibiting STIP1.

We present the following article in accordance with the ARRIVE reporting checklist (available at http://dx.doi. org/10.21037/tcr-20-3333).

\section{Methods}

\section{Cell cultures}

Ovarian cancer cells (MDAH2774, TOV21G and ES2), cervical cancer cells (HeLa), breast cancer cells (MCF7), and lung adenocarcinoma cells (CL1-0) lines were obtained from the American Type Culture Collection (Manassas, VA, USA). Human HAP1 and STIP1 knockout HAP1 cell lines were purchased from Horizon Discovery (Campus Vienna Biocenter 3; Vienna, Austria). Noncancerous cells-immortalized human endometrial stromal Cells (HESC) were purchased from Applied Biological Materials Inc. (abm) (Viking Way, Richmond, BC, V6V 2J5, Canada). Nasopharyngeal cancer cells (NPC-BM1), lung adenocarcinoma cells (CL1-0), colon cancer (HT29), tongue cancer cells (SAS), hepatocellular carcinoma cells (Huh7), and pancreatic cancer cells (BxPC3) were kindly provided by Dr. Chih-Ching Wu (Department of Medical Biotechnology and Laboratory Science, Chang Gung University, Taoyuan, Taiwan) whereas the luciferaseexpressing murine ovarian cancer cells (MOSEC/Luc) was a gift from Dr. Chi-Long Chang (Institute of Biomedical Sciences, Mackay Medical College, Taiwan). MDAH2774, TOV21G, ES2, HeLa, MCF7, CL1-0 were cultured in Dulbecco's Modified Eagle Medium (DMEM/F12 medium). BxPC3 and NPC-BM1, HT-29, MOSEC/ Luc were grown in the Roswell Park Memorial Institute Medium (RPMI 1640 medium). SAS and Huh7 were maintained in the DMEM. Human HAP1 and STIP1 knockout HAP1 cells were cultured in Iscove-modified Dulbecco's medium (IMEM medium). HESC were cultured in RPMI-1640 medium. All media were supplemented with $10 \%$ fetal bovine serum (FBS) and 1\% penicillin and streptomycin, and were obtained from Invitrogen (Life Technologies, Carlsbad, CA, USA).

\section{Antibodies, peptides, and chemicals}

The following antibodies were used in the study: Alexa Fluor 546 anti-rabbit IgG (Thermo Scientific), Alexa Fluor 488 anti-mouse IgG (Thermo Scientific), glyceraldehyde3 -phosphate dehydrogenase (GAPDH), STIP1 and $\beta$-actin (Santa Cruz Biotechnology, Dallas, TX, USA), cleaved caspase-3, cleaved poly (ADP-ribose) polymerase (cleaved PARP) (Cell Signaling Technology, Danvers, MA, USA), LSD1 (Cell Signaling Technology, Danvers, MA, USA) and JAK2 (Abcam, Cambridge, UK). Peptide 520 (STIP1 $1_{520-543}$ : EHLKNPVIAQKIQKLMDVGLIAIR) and peptide 520 containing (D-arginine $)_{8}$ were synthesized and purchased from GeneDireX (Las Vegas City, NE, USA) (23). Scramble peptide was also synthesized from GeneDireX according to the amino acid hydrophobicity of peptide 520. HEPES was obtained from Sigma-Aldrich (St. Louis, MO, USA). 4', 
6'-diamidino-2-phenylindole (DAPI) solution was obtained from Thermo Fisher Scientific (Waltham, MA, USA).

\section{HEPES-mediated intracellular protein delivery}

Cells $\left(8 \times 10^{5}\right)$ were plated in $6-\mathrm{cm}$ dishes for $24 \mathrm{~h}$. The culture media were subsequently replaced by Opti-MEM (Thermo Scientific, Waltham, MA, USA). Different proteins $(7 \mu \mathrm{g})$, including antibodies and small peptides, were mixed with $400 \mu \mathrm{L}$ of a pure HEPES (20 or $30 \mathrm{mM}$; $\mathrm{pH}$ 7.4) solution or Opti-MEM (negative control) for 15-30 $\mathrm{min}$ at room temperature. The transfection mixture was added to cultured cells in Opti-MEM without serum and incubated at $37^{\circ} \mathrm{C}$ for $4-24 \mathrm{~h}$. Briefly, the proteins and HEPES were mixed and incubated for $15 \mathrm{~min}$, and the transfected proteins were detected using fluorescent microscopy after $24 \mathrm{~h}$. The amount of individual components in the transfection mixtures was adjusted according to the size of the cultures. For example, when using a 6-well plate, $4 \mu \mathrm{g}$ of protein were mixed with 200 $\mu \mathrm{L}$ of HEPES (25).

\section{Immunofluorescent staining}

After protein transfection, the cells in culture dishes were washed three times with phosphate-buffered saline (PBS) and fixed with $4 \%$ paraformaldehyde for $20 \mathrm{~min}$. After washing with PBS, the cells were permeabilized with $0.1 \%$ Triton X-100 for $5 \mathrm{~min}$ and blocked with $10 \%$ bovine serum albumin for $30 \mathrm{~min}$. In experiments involving Alexa Fluor 488 anti-mouse IgG (Thermo Scientific), the cells were stained with DAPI for $10 \mathrm{~min}$. After DAPI staining, the slides were washed with PBS three times for $5 \mathrm{~min}$. Fixed cell images were acquired using a confocal microscope with $63 \times$ object lens at excitation and emission wavelengths of 488 and 500-535 nm, respectively (Leica, Wetzlar, Germany).

\section{Western blot analysis}

Cells were lysed in a combination of radioimmunoprecipitation assay buffer $(150 \mathrm{mM} \mathrm{NaCl}, 20 \mathrm{mM}$ Tris- $\mathrm{HCl}(\mathrm{pH} 7.5), 1 \%$ Triton X-100, 1\% NP-40, 0.1\% sodium dodecyl sulphate (SDS), and $0.5 \%$ deoxycholate) and protease/phosphatase inhibitors (Bionovas, Toronto, Canada). The Bradford assay (Bio-Rad, Hercules, CA, USA) was used to determine the total protein concentration. Each sample $(50 \mu \mathrm{g}$ of proteins for each) was subjected to SDS-polyacrylamide gel electrophoresis
(SDS-PAGE). The resolved proteins were transferred onto a nitrocellulose membrane and labeled proteins were visualized using an enhanced chemiluminescence assay (Millipore, Bedford, MA, USA).

\section{Cell viability and cytotoxicity assays}

Different human cancer cell lines were cultured on 96-well plates, and either (I) subjected to HEPES-mediated intracellular delivery of anti-STIP1 (Abnova; Cat. No: H00010963-M02), (Abnova; Cat. No: H00010963-M35) or control IgG2 (Abnova; Cat. No: PAB14685-A01P) antibodies, or (II) exposed to cell-penetrating peptide 520 (23). Following incubation for $24 \mathrm{~h}$, the cells were centrifuged at $300 \times g$ for $5 \mathrm{~min}$, the supernatant was discarded, and the cells attached to the plates were analyzed using a commercially available [3-(4,5-Dimethylthiazol-2-yl)-2,5-diphenyltetrazolium bromide] (MTT) assay (Promega, Madison, WI, USA). Cell toxicity was assessed using the lactate dehydrogenase $(\mathrm{LDH})$ method (Sigma-Aldrich) (25). Since the MTT assay is more sensitive than the LDH assay $(26,27)$, the cells subjected to transfection were incubated for $48 \mathrm{~h}$ and centrifuged at 300 $\mathrm{xg}$ for $5 \mathrm{~min}$. The resulting supernatants were collected and analyzed using a commercially available LDH assay. The MTT and LDH signals were quantified using an enzymelinked immunosorbent assay reader (Tecan Systems Inc., San Jose, CA, USA).

\section{Animal experiments}

Ten-week-old female immunocompetent C57BL/6 mice were about $20 \mathrm{~g}$ and obtained from the National Animal Center (Taipei, Taiwan) and all animal experiment procedures carried out in Laboratory Animal Center, Linkou Chang Gung Memorial Hospital (Taoyuan, Taiwan). Mice were intraperitoneally injected with stably luciferase-expressed C57BL/6 MOSEC/Luc cells (quantity: $\left.1 \times 10^{6}\right)$. C57BL/6 mice bearing MOSEC/Luc cells were randomly divided into two groups. Each treatment group consisted of 4 mice. After one-week, anti-STIP1 or control IgG antibodies pre incubated with $30 \mathrm{mM}$ HEPES (100 $\mu \mathrm{g}$ antibodies $/ 50 \mu \mathrm{L} 30 \mathrm{mM}$ HEPES) for 15 minutes then intravenously injected three times per week until sacrifice. The mice were sacrificed and the presence of tumors and ascites was determined. D-luciferinthe luciferase substrate-was injected intraperitoneally (75 mg/kg body weight) to monitor experimental tumor growth over time. Bioluminescent tumor images were 
visualized on an IVIS imaging system (Xenogen Corp., Alameda, CA, USA) and analyzed with the Living Image software (Xenogen Corp.).

Experiments were performed under a project license (NO.: 2019062502) granted by the Institutional Animal Care and Use Committee of the Chang Gung Memorial Hospital, in compliance with the tenets of the Guide for the Care and Use of Laboratory Animals published by the US National Institutes of Health (NIH Publication No. 85-23, revised 1996).

\section{Statistical analysis}

Continuous data are presented as the mean \pm standard error of the mean (SEM). The results were compared using Student's $t$-test or the Mann-Whitney $U$ test, as appropriate. All calculations were performed in Graph Pad Prism software (version, 5.01; Graph Pad Inc., San Diego, CA, USA). Survival curves were plotted with the Kaplan-Meier method and compared with the log-rank test. Two-tailed P values $<0.05$ were considered statistically significant.

\section{Results}

\section{Intracellular delivery of anti-STIP1 antibodies successfully targets STIP1 within cancer cells}

Anti-STIP1 antibodies were delivered into cancer cells using the HEPES method (25) and subsequently stained with Alexa Fluor 488-conjugated secondary antibodies. The intracellular penetration of antibodies induced by HEPES was confirmed in ovarian cancer cells (MDAH2774) using confocal microscopy (Figure 1A). We then investigated the effects of STIP1 in STIP1-deficient HAP1 cells (knockout, KO). STIP1-expressing HAP1 cells (wild-type, WT) exhibited a higher viability and a lower extent of toxicity than STIP1 KO did, confirming the role of STIP1 in tumorigenesis. Cleaved poly ADP-ribose polymerase (PARP) and cleaved caspase 3, two markers of apoptosis, were also upregulated in STIP1 $\mathrm{KO}$ cells (Figure 1B,C). Taken together, these results indicate that the intracellular delivery of anti-STIP1 antibodies successfully targets STIP1, a molecule that promotes the viability of cancer cells.

\section{Intracellular delivery of anti-STIP1 antibodies induces cell deatb in different cancer cell lines}

Anti-STIP1 antibodies were delivered into different STIP1- overexpressing malignant cells, including pancreatic cancer (BxPC3), head and neck cancer (NPC-BM1), lung cancer (CL1-0), breast cancer (MCF7), colon cancer (HT29), and ovarian cancer (ES2 and MDAH2774) cells. The intracellular delivery of anti-STIP1 consistently exerted cytotoxic effects in all the cancer cell lines (Figure 2A,B,C,D,E,F,G). Moreover, we observed an increased expression of the apoptotic marker cleaved caspase-3 in MDAH2774, TOV21G and ES2 cells (Figure $2 H$ ). Taken together, these results indicated that the HEPES-induced intracellular delivery of anti-STIP1 antibodies inhibits human cancer cell line growth. Moreover, an additional second anti-STIP1 antibody (Abnova; Cat. No: H00010963-M35) was used to perform the experiments of STIP1-mediated cell death in ovarian cancer ES2 cells (Figure S1A). In order to make sure that specificity for cancer cells, we used non-cancerous cells-immortalized human endometrial stromal cells (HESC) to perform the experiments of STIP1-mediated cell death. When treated with antiSTIP1 antibody (Abnova; Cat. No: H00010963-M02 and Cat. No: H00010963-M35) in HESC, there was no change in cell viability or cell cytotoxicity (Figure S1B). Therefore, anti-STIP1 antibody has the potential to develop into anticancer treatment due to its specificity towards tumor cells that express higher STIP1 (Figure S1C).

\section{Spontaneous internalization of cell-penetrating peptide 520, an STIP1 inbibitor, inbibits buman cancer cell line growth}

Peptide 520, a (D-arginine) $)_{8}$-tagged STIP $1_{520-543}$ peptide, is capable of spontaneously entering cells and is known to inhibit the viability of ovarian cancer cells (23). Peptide 520 was internalized spontaneously by pancreatic cancer (BxPC3), head and neck cancer (NPC-BM1), hepatocellular carcinoma (Huh7) cells, breast cancer (MCF7), and tongue cancer (SAS) cells (Figure 3). The internalization of peptide 520 reduced viability and increased toxicity in cancer cell lines.

\section{Intracellular delivery of anti-STIP1 antibodies leads to the degradation of two client proteins of STIP1, LSD1 and $\mathcal{F} A K 2$}

STIP1 inhibition is known to promote the catabolism of LSD1 and JAK2 $(22,23)$ and TRIM2 1 (tripartite motifcontaining protein 21 ) has been reported that it mediated degradation of targeted proteins (28). We performed the intracellular delivery of anti-STIP1 antibodies into TRIM21-expressing HeLa cells consistently decreased 

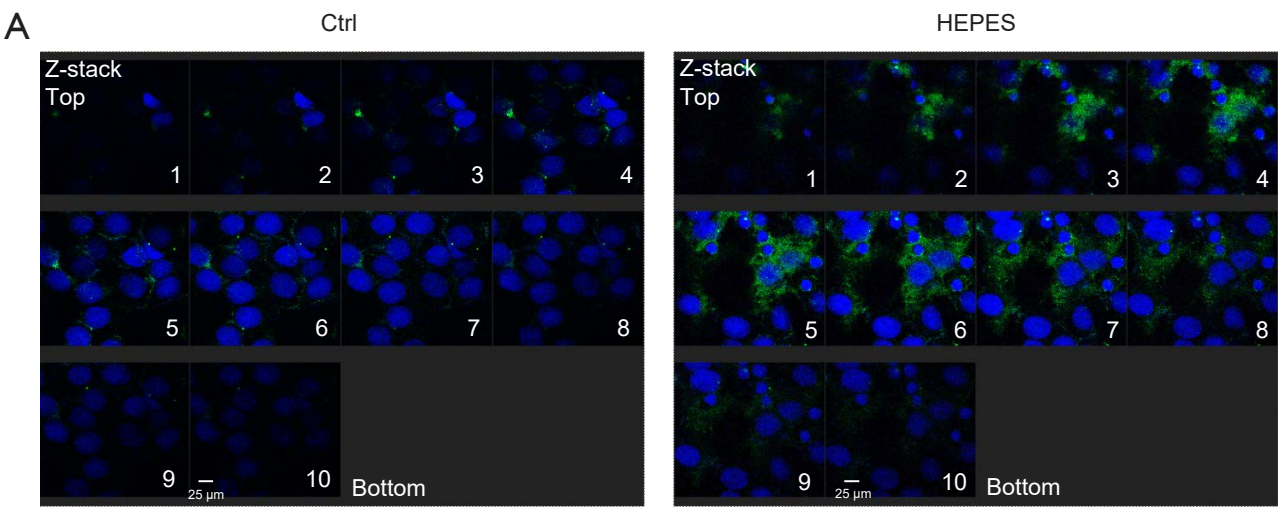

B
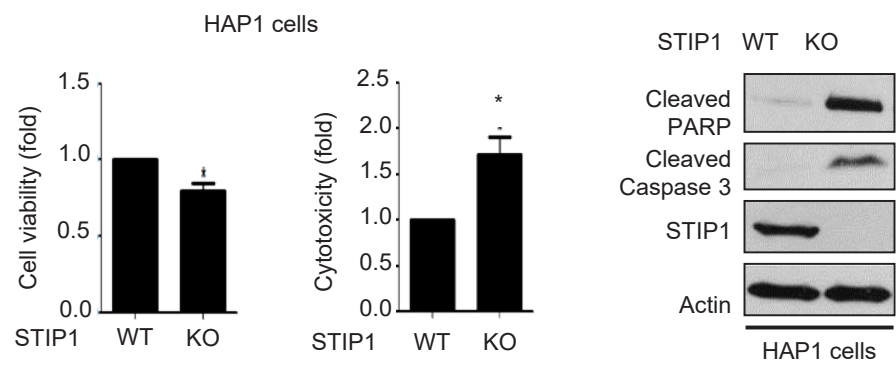

Figure 1 Intracellular stress-induced phosphoprotein 1 (STIP1) as a druggable cancer target. (A) Alexa Fluor 488-conjugated anti-mouse IgG antibodies were transfected in MDAH 2774 cells using either Opti-MEM (Ctrl) or a pure 20-mM HEPES solution. After $24 \mathrm{~h}$ of incubation, the cells were stained with DAPI for $10 \mathrm{~min}$. After DAPI staining, the slides were washed with PBS three times for 5 min, cell images were acquired using a confocal microscope with $63 \times$ object lens at excitation and emission wavelengths of 488 and $500-535 \mathrm{~nm}$, respectively (Leica, Wetzlar, Germany). The fluorescent signals were detected by confocal microscopy via z-stack acquisition. A marked increase in cytosolic fluorescence was observed in the transfection experiments conducted using HEPES solution. Scale bars indicate $25 \mu \mathrm{m}$. (B) STIP1-expressing (WT) or STIP1- knock-out (KO) HAP1 cells were cultured in 96 well plates for $24 \mathrm{~h}$. The cells were collected and analyzed using [3-(4,5-Dimethylthiazol-2-yl)-2,5-diphenyltetrazolium bromide] (MTT) (left panel) and lactate dehydrogenase (LDH) (right panel) assays, respectively. (C) STIP1-expressing (WT) or STIP1-KO HAP1 cells were cultured for $24 \mathrm{~h}$. The cell lysates were then collected and the levels of apoptotic markers (cleaved poly (ADP-ribose) polymerase, cleaved PARP) and cleaved caspase 3 were quantified in the STIP-expressing (WT) or STIP1-KO cells by western blotting. Anti-STIP1 antibodies (Santa Cruz Biotechnology, Dallas, TX, USA) were used to confirm the suppression of STIP1 in STIP1-KO HAP1 cells, whereas antibodies against actin served as controls (housekeeping). Error bars indicate the standard errors of the mean $(\mathrm{n}=3)$. ${ }^{*} \mathrm{P}<0.05$.

the expression of STIP1 and its client proteins, LSD1 and JAK2 (Figure 4). Taken together, these results indicated that the intracellular delivery of anti-STIP1 antibodies can alter STIP1 downstream oncogenic signaling.

\section{In vivo administration of anti-STIP1 antibodies reduces mortality in mice bearing experimental tumors.}

To assess the feasibility of targeting STIP1 in vivo, C57BL/6 mice were intraperitoneally injected with stably luciferaseexpressing C57BL/6 MOSEC/Luc cells followed by the intravenous administration of either anti-STIP1 antibodies or control IgG antibodies-HEPES mixture $(100 \mu \mathrm{g}$ antibodies $/ 50 \mu \mathrm{L} 30 \mathrm{mM}$ HEPES). The results revealed that intravenous administration of anti-STIP1 antibodies group decreased tumor size and reduced mortality in mice bearing experimental tumors $(\mathrm{P}=0.06$; log-rank test; Figure $5 A, B)$.

\section{Discussion}

The results of our study demonstrated that the intracellular targeting of STIP1, attained either via the HEPES- 
A

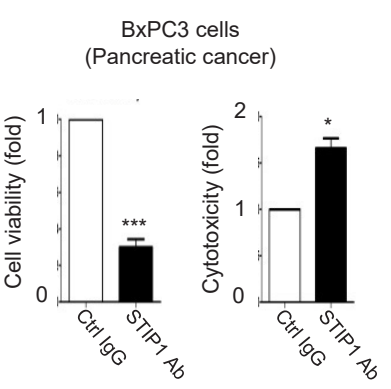

D

MCF7 cells (Breast cancer)
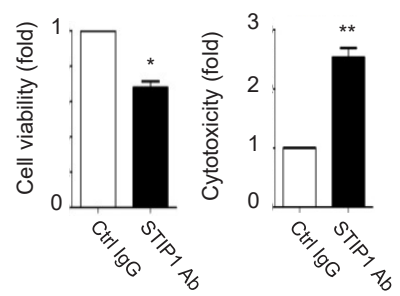

G

MDAH2774 cells (Ovarian cancer)

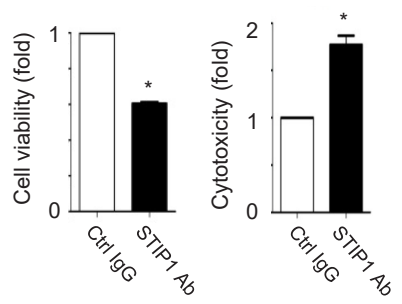

B

NPC-BM1 cells (Head and neck cancer)

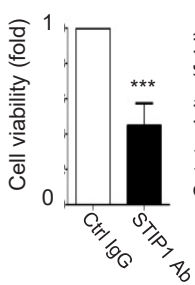

E

HT29 cells (Colon cancer)
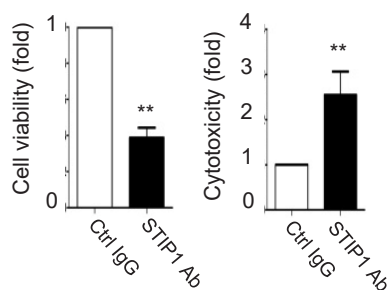

C CL1-0 cells (Lung cancer)
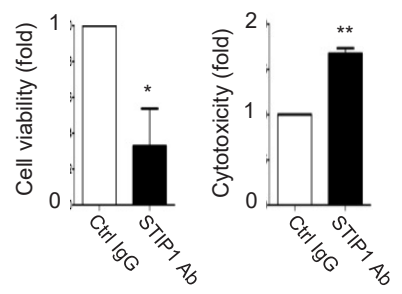

$\mathrm{F}$ ES2 cells (Ovarian cancer)
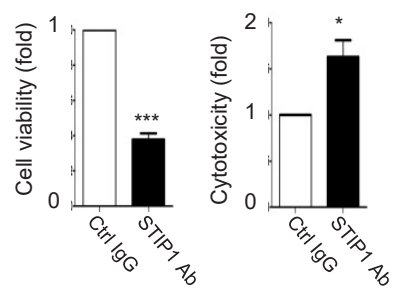

$\mathrm{H}$

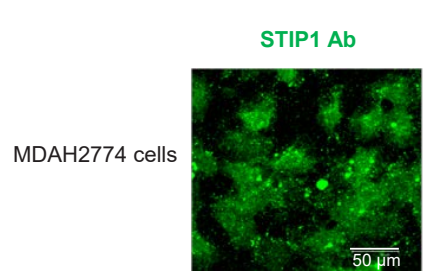

Cleaved caspase 3

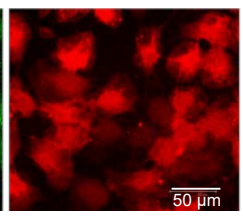

TOV21G cells
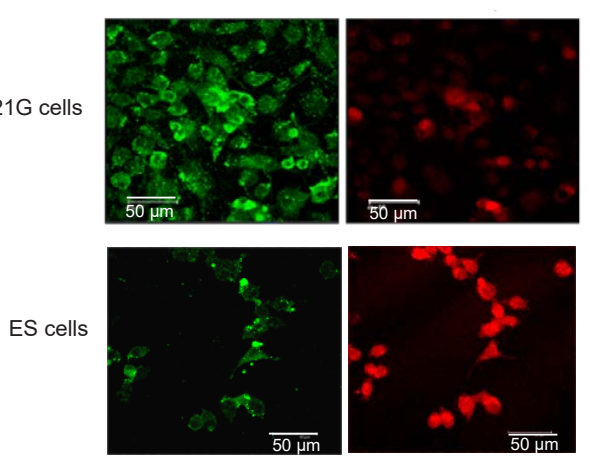

Figure 2 HEPES-mediated intracellular delivery of anti-stress-induced phosphoprotein 1 (STIP1) antibodies inhibits viability and increases toxicity in different cancer cell lines. Control IgG2 antibodies (Ctrl IgG) or anti- STIP1 antibodies (STIP1 Ab) (Abnova; Cat. No: H00010963-M02) were initially mixed with appropriate concentrations of HEPES (reported in parentheses) 15 min before intracellular delivery. Antibodies were subsequently delivered into (A) pancreatic cancer BxPC3 (30 mM HEPES), (B) head and neck cancer NPC-BM1 (20 mM HEPES), (C) lung cancer CL1-0 (20 mM HEPES), (D) breast cancer MCF7 (20 mM HEPES), (E) colon cancer HT29 (20 mM HEPES), (F) ovarian cancer ES2 (20 mM HEPES), and (G) ovarian cancer MDAH2774 (20 mM HEPES) cells. After 24-48 h of incubation, cell viability $(24 \mathrm{~h})$ and cytotoxicity $(48 \mathrm{~h})$ were analyzed with the [3-(4,5-Dimethylthiazol-2-yl)-2,5-diphenyltetrazolium bromide] (MTT) and lactate dehydrogenase (LDH) assays, respectively. (H) The detection of anti-STIP1 antibodies in MDAH2774, TOV21G and ES2 cells were performed by staining with Alexa Fluor 488 anti-mouse IgG antibodies, whereas cleaved caspase 3 antibodies were imaged using Alexa Fluor 546 anti-rabbit IgG antibodies. Scale bars indicate $25 \mu \mathrm{m}$. Error bars indicate the standard errors of the mean $(\mathrm{n}=3)$. ${ }^{*} \mathrm{P}<0.05$, ${ }^{* *} \mathrm{P}<0.01$, and ${ }^{* * *} \mathrm{P}<0.001$. 
A

BxPC3 cells

(Pancreatic cancer)
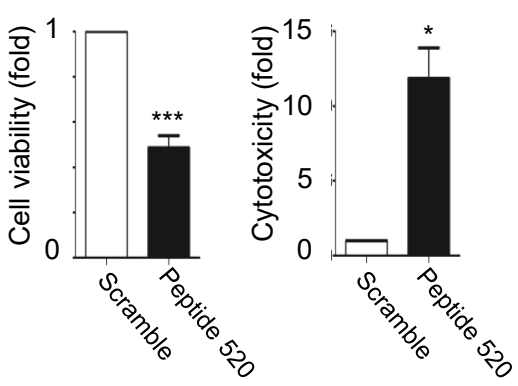

D MCF7 cells (Breast cancer)
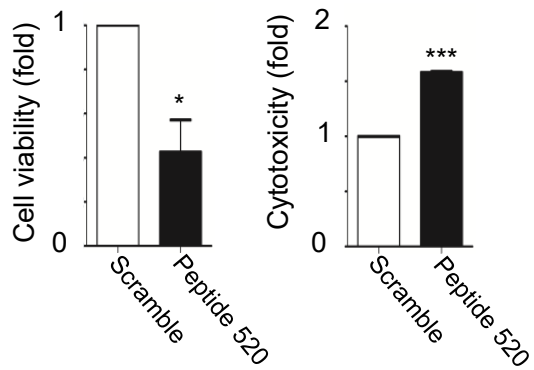

E
B

NPC-BM1 cells (Head and neck cancer)
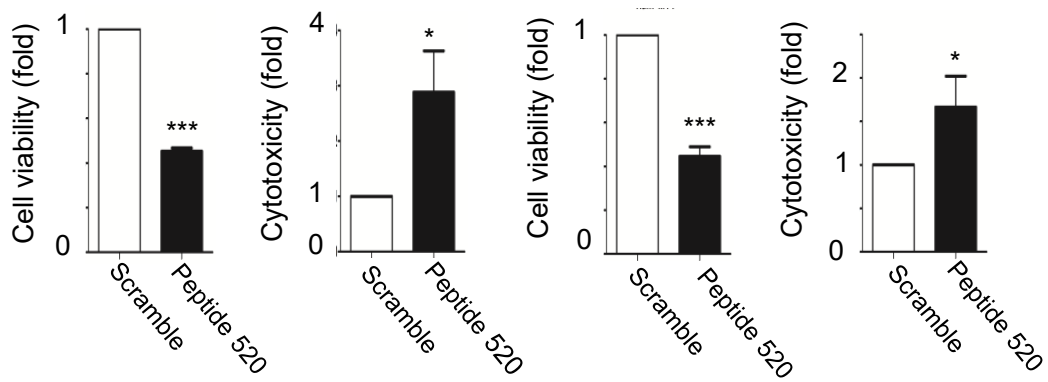

SAS cells (Tongue carcinoma)
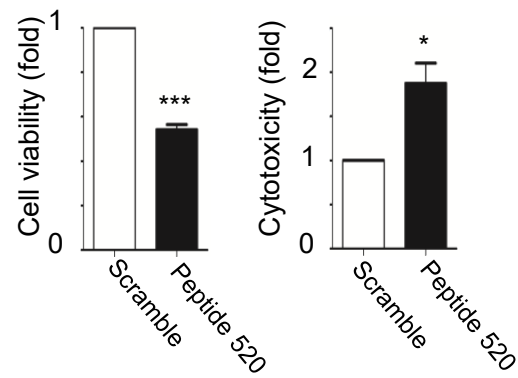

Figure 3 Spontaneous internalization of cell-penetrating peptide 520 inhibits viability and increases toxicity in different cancer cell lines. Peptide 520 underwent spontaneous internalization into (A) pancreatic cancer BxPC3, (B) head and neck cancer NPC-BM1, (C) hepatocellular carcinoma Huh7, (D) breast cancer MCF7, and (E) tongue carcinoma SAS cells with culture medium. A scramble peptide was used as negative control. After $24-48 \mathrm{~h}$ of incubation, cell viability $(24 \mathrm{~h})$ and cytotoxicity (48 h) were analyzed using [3-(4,5-Dimethylthiazol-2-yl)-2,5-diphenyltetrazolium bromide] (MTT) and lactate dehydrogenase (LDH) assays, respectively. Error bars indicate the standard errors of the mean $(\mathrm{n}=3) .{ }^{*} \mathrm{P}<0.05$ and ${ }^{* * *} \mathrm{P}<0.001$.

mediated cytosolic delivery of anti-STIP 1 antibodies or the use of an inhibitory peptide, effectively inhibits human cancer cell line growth. Mechanistically, the intracellular targeting of STIP1 was found to alter downstream oncogenic signaling pathways (involving LSD1 and JAK2 proteins), ultimately resulting in cell death and apoptosis both in vitro $(22,23)$. The detection of anti-STIP 1 antibodies in cancer cells was demonstrated by staining of Alexa Fluor 488 anti-mouse IgG antibodies. Our data provide a basis for the further investigation of STIP1 as an intracellular cancer target, and support the notion that inhibiting intracellular molecules involved in carcinogenesis may be a valuable antitumor strategy $(4,7,8,29,30)$.

STIP1 has been shown to be secreted in the extracellular space, where it promotes tumor growth and metastasis through the activation of various signaling cascades $(13,21,31)$. However, STIP1 is also expressed in the cytoplasm of neoplastic cells $(22,23,25)$, where it elicits numerous cancer growth signaling pathways, including the formation of the HSP90-JAK2-STAT3 complex, the GSK3 $\beta-L S D 1$ pathway, and the nuclear shuttling of Snail1 $(22,23,25)$. The knockdown of STIP1 in pancreatic, ovarian, or gastric cancers has been shown to decrease cancer cell invasiveness and migration capacity via the inhibition of matrix metalloprotease- 2 and $\mathrm{Wnt} / \beta$-catenin signaling $(12,14,32,33)$, as well as via the promotion of apoptotic cell death (34). Interestingly, the intracellular targeting of STIP1 in our study led to an increased expression of apoptotic markers (cleaved PARP and cleaved caspase 3) in STIP1 KO HAP-1 cells. 
A

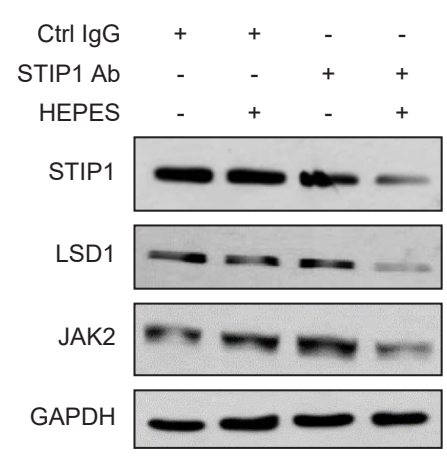

B

C
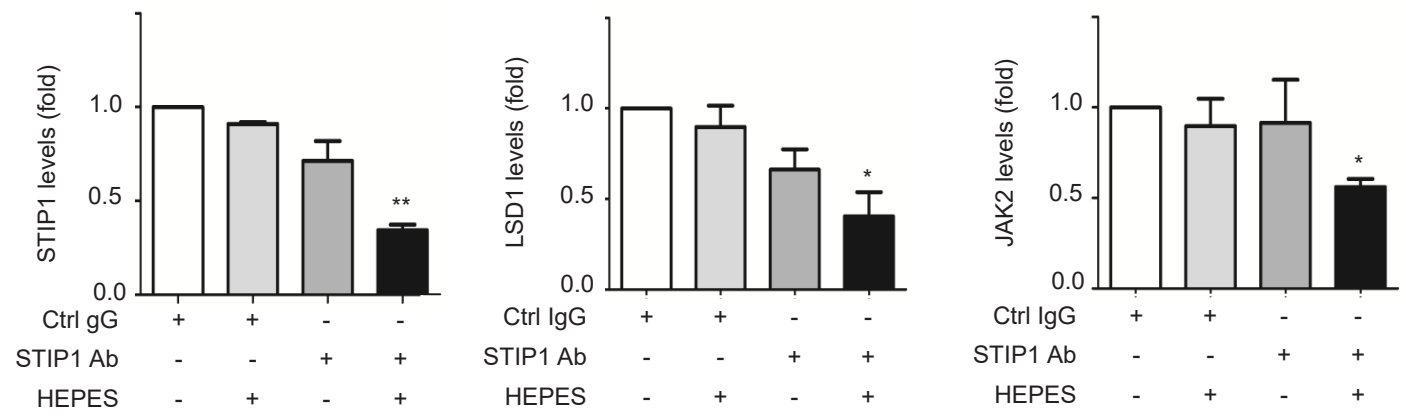

Figure 4 HEPES-mediated intracellular delivery of anti-stress-induced phosphoprotein 1 (STIP1) antibodies reduces STIP1, lysine-specific demethylase 1 (LSD1), and Janus kinase 2 (JAK2) protein levels. (A) Control IgG2 antibodies (Ctrl IgG) or anti-STIP1 antibodies (Abnova; Cat. No: H00010963-M02) were mixed with a pure 30-mM HEPES solution for 15 min before being applied to HeLa cells. After 24 h of incubation, STIP1 (Santa Cruz Biotechnology, Dallas, TX, USA), LSD1, JAK2, and glyceraldehyde-3-phosphate dehydrogenase (GAPDH) (control) protein levels were analyzed by western blotting using the corresponding antibodies. The levels of (B) STIP1, (C) LSD1, and (D) JAK2 proteins were quantified and summarized in a bar graph. Error bars indicate the standard errors of the mean ( $=3$ ). ${ }^{*} \mathrm{P}<0.05$ and ${ }^{* *} \mathrm{P}<0.01$.

Traditional approaches used for the targeting of intracellular molecules as an anticancer strategy include the use of signal molecules fused with peptides (7). Human serum albumin, nanoparticles, and gelatin-based polymers are useful vehicles to facilitate the intracellular delivery of therapeutic antibodies (5). In this study, we demonstrated that the use of HEPES is suitable for promoting the penetration of a therapeutic antibody into the cytosol of malignant cells. These results contribute to paving the way for a future large-scale adoption of this methodological approach in preclinical studies of antibodybased intracellular therapeutic modalities in cancer. As an alternative approach to the intracellular delivery of STIP1 antibodies, the use of a cell-penetrating inhibitory peptide is effective at targeting STIP1 in malignant cells. We have previously shown that (D-arginine) $)_{8}$-tagged peptide 520 is able to enter the cytosol of ovarian cancer cells and block cell growth by disrupting the oncogenic HSP90-JAK2STAT3 signaling pathway (23). Here, we expanded our previous findings by showing that peptide 520 effectively inhibits the in vitro growth of numerous other cancer cell lines from pancreatic, head and neck, breast, tongue, and liver malignancies.

Although we cannot rule out that anti-STIP1 antibodies can bind to STIP1 expressed on the cell surface, we believe that intracellular targeting of STIP1 is of major importance. Furthermore, a head-to-head comparison of the bioavailability of anti-STIP1 antibody and peptide 520 for targeting intracellular STIP1 should be addressed in future investigations. The role of STIP1 expression in different malignancies is also needed to determine the sitespecificity of the tumor response. 
A
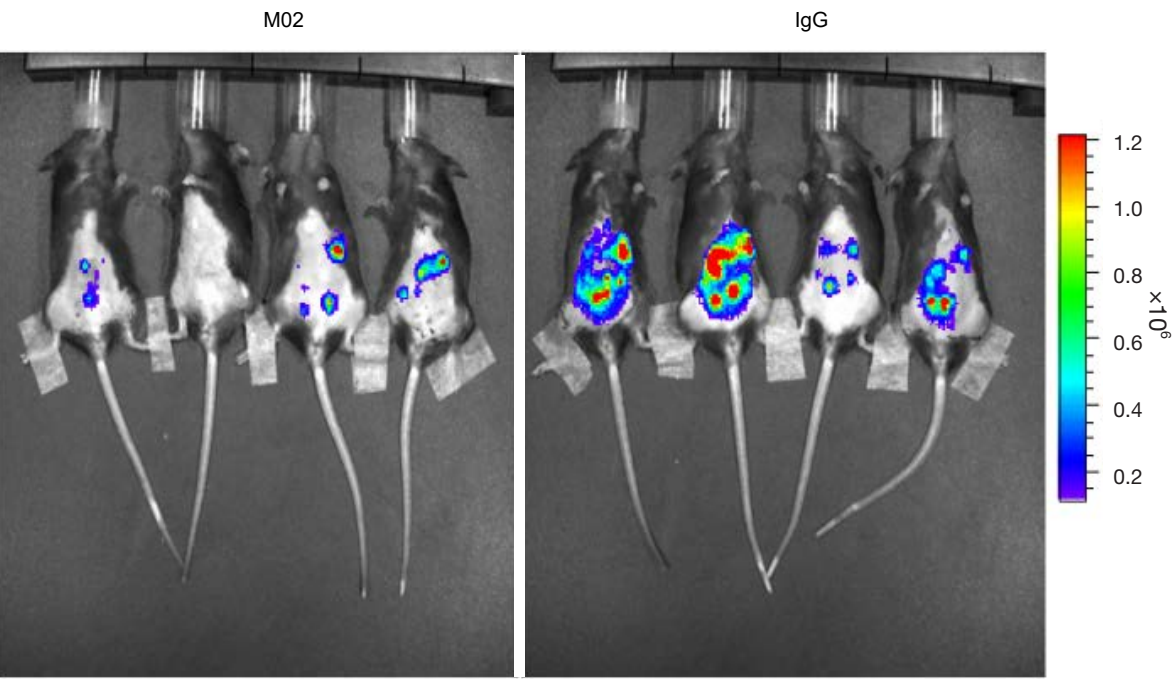

B

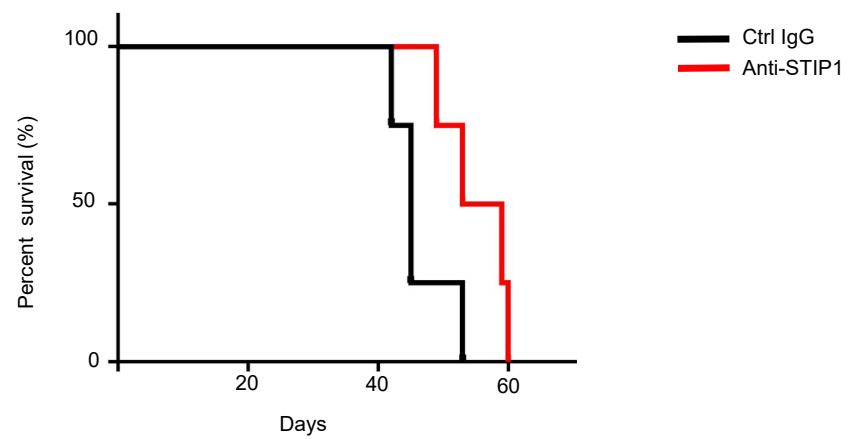

Figure 5 In vivo administration of anti-stress-induced phosphoprotein 1 (STIP1) antibodies reduces mortality in mice bearing experimental tumors. (A) C57BL/6 mice were intraperitoneally injected with malignant cells $\left[1 \times 10^{6}\right.$ luciferase-expressing murine ovarian cancer cells (MOSEC/Luc)] to induce the formation of experimental tumors. After one week, animals underwent intravenous administration of either control mouse IgG2 antibodies (Ctrl IgG) or anti-STIP1 antibodies (M02) (Abnova; Cat. No: H00010963-M02) three times per week (100 $\mu \mathrm{g}$ antibodies $/ 50 \mu \mathrm{L} 30 \mathrm{mM}$ HEPES). Bioluminescent images of animals in the two groups were obtained using an IVIS imaging system. (B) Administration of anti-STIP1 antibodies improved survival compared with Ctrl IgG, $\mathrm{P}=0.06$ (log-rank test).

Further preclinical research efforts are required to fully unravel the complexity of therapeutic responses of cancer cells to intracellular STIP1 inhibition.

In conclusion, our results provide a basis for further investigation of STIP1 as a promising anti-tumor therapeutic strategy. We demonstrated the feasibility of targeting intracellular STIP1 using anti-STIP1 antibody or peptide 520 as a potential anticancer strategy.

\section{Acknowledgments}

The authors would like to express their gratitude to the Microscopy Core Laboratory and the Genomic Medicine
Core Laboratory and Laboratory Animal Center of Chang Gung Memorial Hospital for sharing their expertise.

Funding: This work was financially supported by grants from the Chang Gung Medical Research Foundation (AC: CMRPG3J1301/2/3; CLT: CMRPG3J1381; CYL: CMRPG3J1611) and Taiwan's Ministry of Science and Technology (AC: 108-2314-B-182-031; CLT: 108-2314-B182-033).

\section{Footnote}

Reporting Checklist: The authors have completed the ARRIVE reporting checklist. Available at http://dx.doi. 
org/10.21037/tcr-20-3333

Data Sharing Statement: Available at http://dx.doi. org/10.21037/tcr-20-3333

Conflicts of Interest: All authors have completed the ICMJE uniform disclosure form (available at http://dx.doi. org/10.21037/tcr-20-3333). The authors have no conflicts of interest to declare.

Ethical Statement: The authors are accountable for all aspects of the work in ensuring that questions related to the accuracy or integrity of any part of the work are appropriately investigated and resolved. Experiments were performed under a project license (NO.: 2019062502) granted by the Institutional Animal Care and Use Committee of the Chang Gung Memorial Hospital, in compliance with the tenets of the Guide for the Care and Use of Laboratory Animals published by the US National Institutes of Health (NIH Publication No. 85-23, revised 1996).

Open Access Statement: This is an Open Access article distributed in accordance with the Creative Commons Attribution-NonCommercial-NoDerivs 4.0 International License (CC BY-NC-ND 4.0), which permits the noncommercial replication and distribution of the article with the strict proviso that no changes or edits are made and the original work is properly cited (including links to both the formal publication through the relevant DOI and the license). See: https://creativecommons.org/licenses/by-nc-nd/4.0/.

\section{References}

1. Leader B, Baca QJ, Golan DE. Protein therapeutics: a summary and pharmacological classification. Nat Rev Drug Discov 2008;7:21-39.

2. Smaglo BG, Aldeghaither D, Weiner LM. The development of immunoconjugates for targeted cancer therapy. Nat Rev Clin Oncol 2014;11:637-48.

3. Weiner LM, Murray JC, Shuptrine CW. Antibody-based immunotherapy of cancer. Cell 2012;148:1081-4.

4. Guo K, Li J, Tang JP, et al. Targeting intracellular oncoproteins with antibody therapy or vaccination. Sci Transl Med 2011;3:99ra85.

5. Yin L, Yuvienco C, Montclare JK. Protein based therapeutic delivery agents: Contemporary developments and challenges. Biomaterials 2017;134:91-116.

6. Battogtokh G, Cho YY, Lee JY, et al. Mitochondrial-
Targeting Anticancer Agent Conjugates and Nanocarrier Systems for Cancer Treatment. Front Pharmacol 2018;9:922.

7. Jameson KL, Mazur PK, Zehnder AM, et al. IQGAP1 scaffold-kinase interaction blockade selectively targets RASMAP kinase-driven tumors. Nat Med 2013;19:626-30.

8. Dao T, Yan S, Veomett N, et al. Targeting the intracellular WT1 oncogene product with a therapeutic human antibody. Sci Transl Med 2013;5:176ra33.

9. Schmid AB, Lagleder S, Grawert MA, et al. The architecture of functional modules in the Hsp90 cochaperone Sti1/Hop. Embo J 2012;31:1506-17.

10. Blair LJ, Genest O, Mollapour M. The multiple facets of the Hsp90 machine. Nat Struct Mol Biol 2019;26:92-5.

11. Sun W, Xing B, Sun Y, et al. Proteome analysis of hepatocellular carcinoma by two-dimensional difference gel electrophoresis: novel protein markers in hepatocellular carcinoma tissues. Mol Cell Proteomics 2007;6:1798-808.

12. Walsh N, O'Donovan N, Kennedy S, et al. Identification of pancreatic cancer invasion-related proteins by proteomic analysis. Proteome Sci 2009;7:3.

13. Wang TH, Chao A, Tsai CL, et al. Stress-induced phosphoprotein 1 as a secreted biomarker for human ovarian cancer promotes cancer cell proliferation. Mol Cell Proteomics 2010;9:1873-84.

14. Chao A, Lai CH, Tsai CL, et al. Tumor stress-induced phosphoprotein1 (STIP1) as a prognostic biomarker in ovarian cancer. PLoS One 2013;8:e57084.

15. Kubota $H$, Yamamoto $S$, Itoh $E$, et al. Increased expression of co-chaperone HOP with HSP90 and HSC70 and complex formation in human colonic carcinoma. Cell Stress Chaperones 2010;15:1003-11.

16. Van Simaeys D, Turek D, Champanhac C, et al. Identification of cell membrane protein stress-induced phosphoprotein 1 as a potential ovarian cancer biomarker using aptamers selected by cell systematic evolution of ligands by exponential enrichment. Anal Chem 2014;86:4521-7.

17. Wu R, Liu F, Peng P, et al. Tumor stress-induced phosphoprotein 1 as a prognostic biomarker for breast cancer. Ann Transl Med 2018;6:302.

18. Luo X, Liu Y, Ma S, et al. STIP1 is over-expressed in hepatocellular carcinoma and promotes the growth and migration of cancer cells. Gene 2018;662:110-7.

19. Zhang S, Shao J, Su F. Prognostic significance of STIP1 expression in human cancer: A meta-analysis. Clin Chim Acta 2018;486:168-76.

20. Zhang Z, Ren H, Yang L, et al. Aberrant expression of 
stress-induced phosphoprotein 1 in colorectal cancer and its clinicopathologic significance. Hum Pathol 2018;79:135-43.

21. Tsai CL, Tsai CN, Lin CY, et al. Secreted stress-induced phosphoprotein 1 activates the ALK2-SMAD signaling pathways and promotes cell proliferation of ovarian cancer cells. Cell Rep 2012;2:283-93.

22. Tsai CL, Chao AS, Jung SM, et al. Stress-induced phosphoprotein 1 acts as a scaffold protein for glycogen synthase kinase-3 beta-mediated phosphorylation of lysine-specific demethylase 1. Oncogenesis 2018;7:31.

23. Tsai CL, Chao A, Jung SM, et al. Stress-induced phosphoprotein-1 maintains the stability of JAK2 in cancer cells. Oncotarget 2016;7:50548-63.

24. Su T, Liao J, Dai Z, et al. Stress-induced phosphoprotein 1 mediates hepatocellular carcinoma metastasis after insufficient radiofrequency ablation. Oncogene 2018;37:3514-27.

25. Chen SH, Chao A, Tsai CL, et al. Utilization of HEPES for Enhancing Protein Transfection into Mammalian Cells. Mol Ther Methods Clin Dev 2018;13:99-111.

26. Fotakis $\mathrm{G}$, Timbrell JA. In vitro cytotoxicity assays: comparison of LDH, neutral red, MTT and protein assay in hepatoma cell lines following exposure to cadmium chloride. Toxicol Lett 2006;160:171-7.

27. Lobner D. Comparison of the LDH and MTT assays for

Cite this article as: $\mathrm{Lin} \mathrm{CY}$, Chen $\mathrm{SH}$, Tsai CL, Tang $\mathrm{YH}, \mathrm{Wu}$ KY, Chao A. Intracellular targeting of STIP1 inhibits human cancer cell line growth. Transl Cancer Res 2021;10(3):1313-1323. doi: $10.21037 /$ tcr-20-3333 quantifying cell death: validity for neuronal apoptosis? J Neurosci Methods 2000;96:147-52.

28. Clift D, McEwan WA, Labzin LI, et al. A Method for the Acute and Rapid Degradation of Endogenous Proteins. Cell 2017;171:1692-706.e18.

29. Veomett N, Dao T, Liu H, et al. Therapeutic efficacy of an Fc-enhanced TCR-like antibody to the intracellular WT1 oncoprotein. Clin Cancer Res 2014;20:4036-46.

30. Dao T, Pankov D, Scott A, et al. Therapeutic bispecific T-cell engager antibody targeting the intracellular oncoprotein WT1. Nat Biotechnol 2015;33:1079-86.

31. Wang J, You H, Qi J, et al. Autocrine and paracrine STIP1 signaling promote osteolytic bone metastasis in renal cell carcinoma. Oncotarget 2017;8:17012-26.

32. Walsh N, Larkin A, Swan N, et al. RNAi knockdown of Hop (Hsp70/Hsp90 organising protein) decreases invasion via MMP-2 down regulation. Cancer Lett 2011;306:180-9.

33. Huang L, Zhai E, Cai S, et al. Stress-inducible Protein-1 promotes metastasis of gastric cancer via Wnt/beta-catenin signaling pathway. J Exp Clin Cancer Res 2018;37:6.

34. Beraldo FH, Soares IN, Goncalves DF, et al. Stressinducible phosphoprotein 1 has unique cochaperone activity during development and regulates cellular response to ischemia via the prion protein. FASEB J 2013;27:3594-607. 


\section{Supplementary}

A
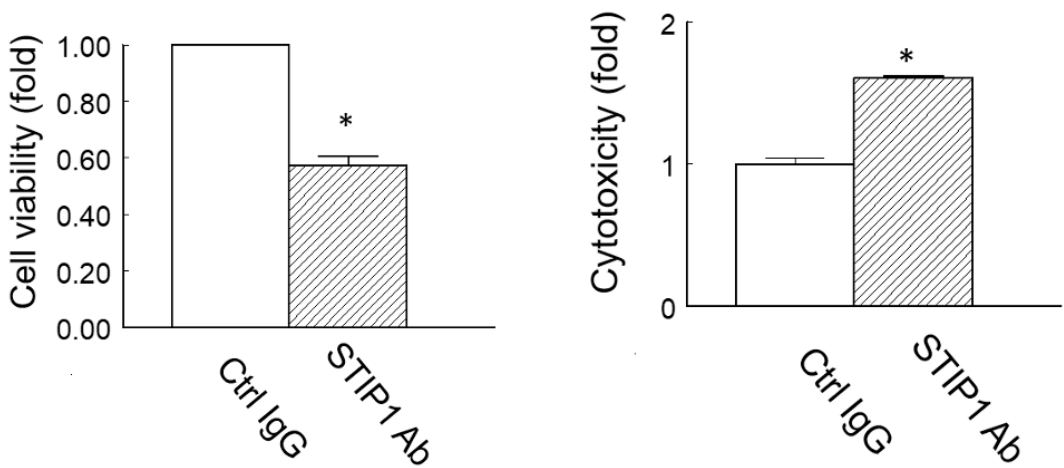

B
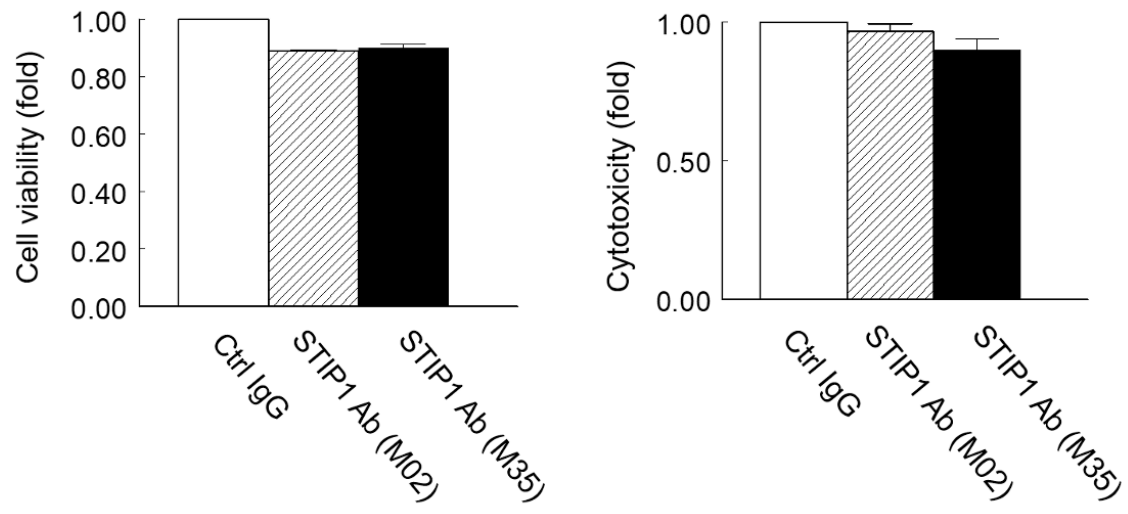

C
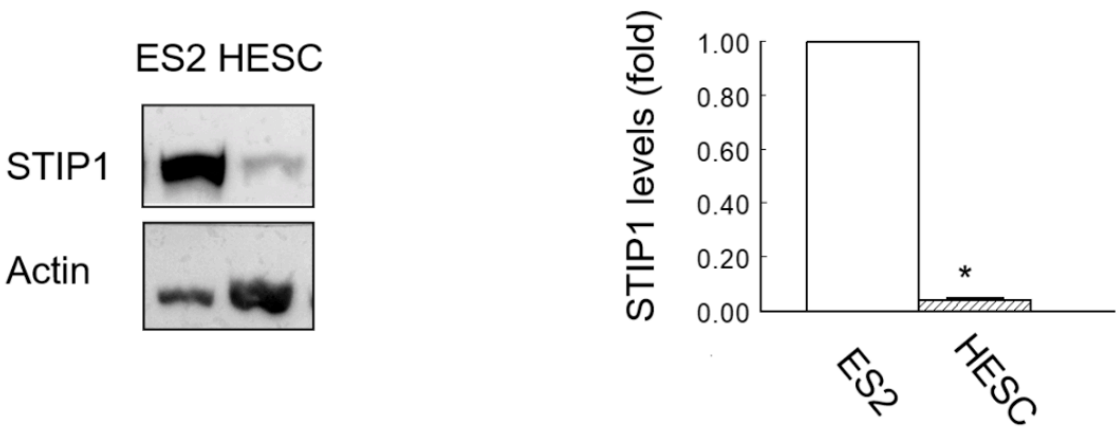

Figure S1 HEPES-mediated intracellular delivery of anti-STIP1 antibodies inhibits viability and increases toxicity in different cell lines. Control IgG2 antibodies (Ctrl IgG), anti-STIP1 antibodies (STIP1 Ab) (Abnova; Cat. No: H00010963-M02): STIP1-(M02) or antiSTIP1 antibodies (STIP1 Ab) (Abnova; Cat. No: H00010963-M35): STIP1-(M35) were initially mixed with 20mM HEPES 15 min before intracellular delivery. Antibodies were subsequently delivered into (A) ovarian cancer ES2 (B) non-cancerous cells-immortalized human endometrial stromal Cells (HESC). After 24-48 h of incubation, cell viability (24 h) and cytotoxicity (48 h) were analyzed with the MTT and LDH assays, respectively. (C) ES2 and HESC cells were cultured for $24 \mathrm{~h}$. The cell lysates were then collected and the level of STIP1 (Santa Cruz Biotechnology, Dallas, TX, USA) was quantified in the ES2 and HESC cells by western blotting. Antibodies against actin served as controls (housekeeping). Error bars indicate the standard errors of the mean $(\mathrm{n}=3)$. ${ }^{*} \mathrm{p}<0.05$. 